\title{
Association of the characteristics of B- and T-cell repertoires with papillary thyroid carcinoma
}

\author{
GUOPING SUN $^{1 *}$, LUMEI QIU ${ }^{2 *}$, ZHIQIANG CHENG ${ }^{2}$, WEIBING PAN $^{1}$, JINGJUN QIU $^{1}$,

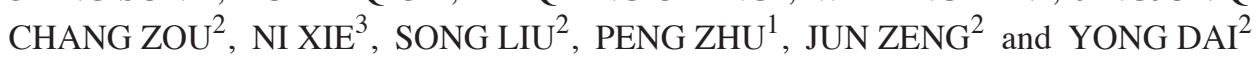 \\ ${ }^{1}$ Core Laboratory, Pingshan New District People's Hospital, Shenzhen, Guangdong 518118; \\ ${ }^{2}$ Clinical Medical Research Center, The Second Clinical Medical College of Jinan University, \\ Shenzhen People's Hospital, Shenzhen, Guangdong 518020; ${ }^{3}$ Core Laboratory, Shenzhen Second People's Hospital, \\ First Affiliated Hospital of Shenzhen University, Shenzhen, Guangdong 518035, P.R. China
}

Received March 18, 2017; Accepted November 16, 2017

DOI: $10.3892 / \mathrm{ol} .2018 .8800$

\begin{abstract}
Papillary thyroid carcinoma (PTC) is the most common type of thyroid cancer.Complementarity-determining region 3 (CDR3) of $\mathrm{B}$-cell receptors (BCRs) and $\mathrm{T}$-cell receptors are the major site of antigen recognition, which determines a unique clone type, and are considered to be the representative of the disease. In the present study, high-throughput sequencing was used to analyze the association of characteristics of the BCR immunoglobulin heavy chain (IGH) and the T-cell receptor $\beta$ chain (TRB) CDR3 genes in PTC and corresponding pericarcinous tissues from patients. A difference of CDR3 length distributions of total IGH CDR3 sequences between the two groups was revealed. IGHV3-11/IGHJ6, TRBV2/TRBJ1-2 and TRBV2/TRBJ1-1 may be biomarkers for the development of PTC. Furthermore, it was revealed that the extent of the common clonotype expressions at the amino acid level was slightly higher compared with the nucleotide level. The Shannon entropy demonstrated a diversity reduction in PTC compared with the pericarcinous group, and the highly expended clone (HEC) expression of PTC was higher compared with that of the corresponding pericarcinous group. Additionally, the highest clone frequency percentage of IGH and TRB was at $0.1-1.0 \%$ degree of expansion, as HEC expression was higher in PTC compared with the matched group. There was no shared clone of HECs in the two groups either at the amino acid level or at
\end{abstract}

Correspondence to: Professor Yong Dai, Clinical Medical Research Center, The Second Clinical Medical College of Jinan University, Shenzhen People's Hospital, 1017 Dongmen North Road, Luohu, Shenzhen, Guangdong 518020, P.R. China

E-mail: daiyong22@aliyun.com

${ }^{*}$ Contributed equally

Key words: papillary thyroid carcinoma, B-cell receptors, T-cell receptors, complementarity-determining region 3 , high-throughput sequencing the nucleotide expression level. The differential expression of CDR3 sequences of PTC have been identified in the present study. Further research is required for assessing the immune repertoire size, diversity, cloning tracking and finding public clones of T-cell and B-cell populations in the development of PTC.

\section{Introduction}

Thyroid cancer accounts for $\sim 1 \%$ of all malignancies globally in 2003, including papillary thyroid carcinoma (PTC), follicular thyroid carcinoma, poorly differentiated thyroid carcinoma, medullary thyroid carcinoma and anaplastic thyroid carcinoma (1). Of these, PTC is the most common type of thyroid cancer (2). Furthermore, the Chinese Cancer Registry reported that the incidence of thyroid carcinoma is 6.6 out of 100,000 individuals in China in 2011 (3). Of all patients with thyroid carcinoma, $80 \%$ have PTC, which is named for its papillary histological architecture in 2011 (4). Thyroid cancer therapy is based on surgery followed by iodine-131 treatment to avoid potential tumor remnants and metastases. Clinicopathological factors including age, sex, tumor size and lymphadenopathy are risk factors of PTC progression (5). Lymph node metastasis is common in patients with PTC, and it has been identified in $20-50 \%$ of patients prior to the initial treatment of PTC (6). Therefore, further research into the pathogenesis of and therapeutic biomarkers for PTC diagnosis and treatment is worthwhile.

Adaptive immunity, based on its enormous diversity of BCRs and TCRs, may essentially provide acute and long-term protection against a limitless array of pathogenic hazards (7). The immune repertoire is mainly composed of BCRs and TCRs in the process of the dynamic adaptive immune system (7).

BCRs and TCRs are composed of two heavy chains and two light chains. Heavy chains comprise a variable region ( $\mathrm{V}$ region), a constant region (C region), a transmembrane region and a cytoplasmic domain, whilst light chains only have variable regions and constant regions. $\mathrm{V}$ regions are composed of two domains [heavy chains $(\mathrm{VH})$ and light chains (VL) for BCRs, and primarily $\alpha / \beta$ and rarely $\gamma / \delta$ for TCRs], 
which consists of three complementarity determining regions (CDR1, CDR2 and CDR3) (7). During B and T lymphocytes development, variable antigen receptor gene segments [Variable (V), Diversity (D) and Joining (J)] are rearranged through targeted DNA recombination events. This method contributes notably to BCR and TCR specificities which are different in the length of their amino acid sequences $(6,7)$. In total, the diversity established through these molecular mechanisms is substantial, with the estimated theoretical numbers of distinct BCRs and TCRs exceeding $10^{13}$ and $10^{18}$, respectively (7).

Structural differences between each type of specific immune protein are minimal in an immune repertoire (7). Whilst there are numerous different subtypes, this diversity serves a vital function in the maintenance of health. The more the subtypes of specific immune protein exist, the more effective a response against any pathogen. Conversely, the fewer subtypes there are, the more an individual is susceptible to disease (8). In addition, age, environment, drug-induced diseases and multiple other factors also affect the diversity of the immune repertoire (6). The CDR3 region of BCR and TCR is the major recognition site of tumor antigens $(1,7)$; therefore, it may recognize a unique clone type, which represents the disease. Therefore, the clonal diversity of lymphocytes may be judged by detecting the length of CDR3 (7). Based on this, through the study of specific BCR and TCR repertoires in patients with PTC by high throughput sequencing (HTS), degree of cloning and specificity may help to explore B- and T-cells in the occurrence and development of PTC.

HTS, which is alternatively called second-generation sequencing (next generation sequencing), has notably influenced research potential in immunology (9). It is a massive parallel sequencing technology. The cost of HTS has been reduced by producing a large number of short sequences in order to provide a high-throughput read of multiple orders of magnitude (8). HTS technology has revolutionized genomics and genetic research. Illumina released a lower throughput fast-turnaround instrument in 2011, the MiSeq, which was aimed at smaller laboratories and the clinical diagnostic market (9). Sequencing of the whole immune repertoire has become feasible and affordable (8). The existing sequencing with Illumina technology allows $2 \times 150$ base pairs to be run every $24 \mathrm{~h}$ and $2 \times 250$ base pairs to be run every $35 \mathrm{~h}$ using the MiSeq platform. This technology is widely used in small and medium-sized genome research, including influenza virus, human immunodeficiency virus drug resistance testing, acute myeloid leukemia and inherited cardiac disease (data from BGI). As HTS accounted for $>2 / 3$ of the market share, and is supported by a majority of third-party software (10), the widespread application of HTS technology in cancer genomics research will provide novel insights into determining the clonal association in patients with PTC.

\section{Materials and methods}

Patients and controls. Samples of PTC tissues and the corresponding pericarcinoma tissues were collected from 5 patients with PTC from the Shenzhen People's Hospital (Shenzhen, China) between April and October in 2015. The
5 patients were diagnosed with PTC; 3 were males and 2 females, with a mean age of 35.6 years (range, $24-43$ years). The 5 pericarcinoma tissues were matched with the 5 PTC tissues. Immediately following resection, the surgical specimens were analyzed by a pathologist. Preoperative cytology was combined with immunohistochemical diagnosis and postoperatively embedded in paraffin once frozen. The diagnosis of PTC was confirmed by pathological diagnosis and clinical evidence.

Written informed consent for participation in the present study was obtained from all subjects. The present study was approved by the Ethics Committee of Shenzhen People's Hospital and abided by the Helsinki Declaration for medical research involving human subjects.

DNA extraction and mix. DNA was extracted by standard methods, as follows. Dewaxing was performed using xylene and followed by overnight proteinase K (Sigma-Aldrich; Merck KGaA, Darmstadt, Germany) digestion at $65^{\circ} \mathrm{C}$ for pathological paraffin sections. The total genomic DNA samples were then extracted from pathological sections of PTC and pericarcinoma tissues from 5 patients using a QIAamp DNA Mini kit (Qiagen GmbH, Hilden, Germany) according to the manufacturer's protocol. DNA quality was evaluated using $0.8 \%$ agarose gel electrophoresis (120V) and staining with $0.5 \mu \mathrm{g} / \mathrm{ml}$ ethidium bromide at $55^{\circ} \mathrm{C}$ for $20 \mathrm{~min}$ (Sigma-Aldrich; Merck KGaA) and DNA concentration was quantified using a Qubit fluorometer (Thermo Fisher Scientific, Inc., Waltham, MA, USA). The PTC tissue DNA samples were mixed into one specimen according to a 1:1:1:1:1 proportion, named as the PTC sample; the pericarcinoma tissue DNA samples were processed in the same manner, and named the PTC pericarcinoma sample.

Multiplex-polymerase chain reaction (PCR) amplification of the immunoglobulin heavy chain $(I G H)$ and $T$-cell receptor $\beta$ chain (TRB) CDR3 region. The human IGH and TRB sequences were downloaded from IMGT ${ }^{\circledR}$ (http://www.imgt. org/) (11). In general, the initial amount of genomic DNA for one reaction was $500 \mathrm{ng}$ (the lowest concentration was not $<35 \mathrm{ng} / \mu \mathrm{l}$ ), and multiple PCR (30 cycles) were used to amplify the rearranged IGH and TRB CDR3 region by QIAGEN multiplex PCR kit (Qiagen $\mathrm{GmbH}$ ) once specially designed forward and reverse primers (included in the kit) of $\mathrm{V}$ and $\mathrm{J}$ region were added. Primers were combined according to the adaptor sequence and specific gene sequence. The PCR protocol was performed according to standard procedure (12). The amplification protocol was as follows: $15 \mathrm{~min}$ at $95^{\circ} \mathrm{C}$, 30 cycles of $30 \mathrm{sec}$ at $94^{\circ} \mathrm{C}, 30 \mathrm{sec}$ at $59^{\circ} \mathrm{C}$ and $1 \mathrm{~min}$ at $72^{\circ} \mathrm{C}$, followed by a final extension cycle of $10 \mathrm{~min}$ at $72^{\circ} \mathrm{C}$ on a PCR Express thermal cycler (Hybaid; Thermo Fisher Scientific, Inc.). The library was separated on a $0.8 \%$ agarose gel, and the target fragments were isolated and purified by QIAquick Gel Extraction kit (Qiagen $\mathrm{GmbH}$ ) following the enrichment of joint modification of DNA fragments.

HTS. DNA insert fragments in the library were tested using the Agilent 2100 Bioanalyzer (Agilent 1000 Reagents; Agilent Technologies, Inc., Santa Clara, CA, USA), and the concentration was quantified using an ABI StepOnePlus Real-Time PCR 
System (TaqMan Probe; Applied Biosystems; Thermo Fisher Scientific, Inc.). The aforementioned library was qualified by $\mathrm{NaOH}$ according to the protocol (Illumina, Inc., San Diego, CA, USA), diluted with hybridization buffer HT1 (Illumina, Inc.) to a final dilution of $10 \mathrm{pm}$, then subjected to flowcell hybridization $\left(15 \mathrm{~min}\right.$ at $95^{\circ} \mathrm{C}, 30$ cycles of $30 \mathrm{sec}$ at $94^{\circ} \mathrm{C}$, $30 \mathrm{sec}$ at $59^{\circ} \mathrm{C}$ and $1 \mathrm{~min}$ at $72^{\circ} \mathrm{C}$, followed by a final extension cycle of $10 \mathrm{~min}$ at $72^{\circ} \mathrm{C}$ ) and then combined with TruSeq PE Cluster kit v3-cBot-HS reagent (Illumina, Inc.) to complete bridge PCR amplification, according to the manufacturer's protocol. Finally, the prepared flowcell was tested using the MiSeq sequencing.

Data analysis. The raw data was filtered using adapter contamination. The threshold average quality score was $<15$, based on the Illumina 0-41 quality system (12), and a threshold for the proportion of $\mathrm{N}$ bases was $<5 \%$. Bases with a low quality score $(<10)$ were trimmed; the quality score was expected to be $>15$ after trimming and the remaining sequence length was expected to be $>60 \mathrm{nt}$. Subsequent to the above filtering conditions, the filtered clean reads were obtained, and then the two sequences were joined into a complete contig.

The analysis platform introduced by PCR has an automated adjustment to errors and it may reveal IGH and TRB CDR3 cloning expression and indel distribution by sequencing. According to the comparison analysis, the IGH and TRB V(D)J junction region, in addition to the last position of the $\mathrm{V}$ gene, the start site of the $\mathrm{D}$ gene, the end site of the $\mathrm{D}$ gene and the start site of the J gene were completely rearranged to identify $\mathrm{V}(\mathrm{D}) \mathrm{J}$ insertion and deletion bases and the length distribution. In brief, CDR3 region and sequence length distribution were compared between PTC and pericarcinous tissues.

According to the comparison analysis, the expression of each clone, distinct DNA sequence amino acid sequence and the combination of V-J were calculated. The diversity of the sample with respect to each distinct clone and the Shannon entropy based on distinct DNA, amino acids and V-J combination may be calculated (13). The clone expression level of each sample was also calculated at different resolutions of distinct DNA sequence, amino acid sequence and V-J combination. HECs in the present study refer to the CDR3 sequences whose expression quantity were $>0.5 \%$ of the total expression. In general, if the CDR3 sequences of PTC tissues are higher compared with corresponding pericarcinous tissues, it indicates the presence of abnormal amplification CDR3 sequences in patients. Pearson's correlation coefficient was conducted to assess the relevance of IGHV-J and TRBV-J usage ratio between PTC and pericarcinoma groups.

\section{Results}

Summary of sequencing. Using HTS, IGH and TRB repertoires sourced from B- and T-lymphocytes collected from PTC and pericarcinous tissues from 5 patients were sequenced. A total of 486,007 and 745,353 BCR reads were obtained from PTC and corresponding pericarcinoma tissues, respectively. A total of 841,591 and 473,761 TCR reads were additionally obtained from PTC and corresponding pericarcinoma tissues, respectively. Following filtering, 292,683 and 341,209 total BCR CDR3 sequences were acquired from patients and corresponding pericarcinoma tissues, respectively, and 561,607 and 335,334 total TCR CDR3 sequences were acquired from patients and corresponding pericarcinoma tissues, respectively. There were 13,896 and 27,156 unique BCR CDR3 sequences identified at the nucleotide level, which encoded 10,763 and 21,799 sequences at the amino acid level from patients and corresponding pericarcinoma tissues, respectively. Meanwhile, 17,612 and 20,410 unique TCR CDR3 sequences were obtained at the nucleotide level, which encoded 13,669 and 16,789 sequences at the amino acid level from patients and corresponding pericarcinoma tissue, respectively (Table I).

Distribution characteristics of CDR3 length. The length distributions of IGH appear to be different between PTC $\left(\mathrm{R}^{2}=0.7953\right)$ and pericarcinoma $\left(\mathrm{R}^{2}=0.9503\right)$ groups. In the two groups, the 3 most frequently observed CDR3 lengths were 13, 14 and 15 . The PTC group has substantially skewed distribution whilst the paired pericarcinoma group has mainly normal distribution (Fig. 1A). The difference of length distributions of TRB between PTC $\left(\mathrm{R}^{2}=0.9932\right)$ and pericarcinoma $\left(R^{2}=0.9646\right)$ groups was minimal (Fig. 1B).

$V(D) J$ region characteristics and $V-J$ region gene usage of IGH and TRB in patients. To explore the characteristics of $\mathrm{V}(\mathrm{D}) \mathrm{J}$ region in patients with PTC, data was collected and compared with the public database IMGT ${ }^{\circledR}$ (http://www.imgt.org/) (10). IGH and TRB CDR3 peptide sequences were identified according to the characteristics of the $\mathrm{V}(\mathrm{D}) \mathrm{J}$ region. Using HTS, $83 \mathrm{~V}$ genes, $37 \mathrm{D}$ genes and $6 \mathrm{~J}$ genes of IGH CDR3 sequences were obtained. A total of $58 \mathrm{~V}$ genes, $2 \mathrm{D}$ genes and $14 \mathrm{~J}$ genes of TRB CDR3 sequences were obtained.

V-J gene usage frequency, which accurately reflects the key features of CDR3 recognition specificity, also reveals differences between two groups. A total of 275 IGHV-J pairings were identified in PTC and pericarcinoma groups. The most frequent pairing in PTC and pericarcinoma was IGHV3-11/IGHJ6 (Fig. 2A), which accounted for 15.51 and $9.12 \%$ of total pairings, respectively. Pearson's correlation coefficient of pairwise comparison between PTC and pericarcinoma groups of IGHV-J usage ratio was 0.89 (data not shown). On the other hand, there were 536 TRB V-J pairings existing in the two groups. The most frequent pairing among them were TRBV2/TRBJ1-2 and TRBV2/TRBJ1-1 (Fig. 2B) contributing to 5.26 and $5.27 \%$ of total pairings, respectively. Pearson's correlation coefficient of pairwise comparison between PTC and pericarcinoma groups of TRBV-J usage ratio was 0.68 (data not shown).

Analysis of shared sequence at the nucleotide and amino acid level. Amino acid sequences of CDR3, which reveal the function of the immune repertoire, may more accurately reflect the specificity of the combination of lymphocyte and antigen compared with the $\mathrm{V}(\mathrm{D}) \mathrm{J}$ gene. In order to explore the similarity of IGH and TRB sequence libraries, a number of consensus sequences of each paired sample between the two groups were identified. Common IGH CDR3 sequences accounted for $5.03 \%$ at the nucleotide level and $6.57 \%$ at the 
Table I. BCR and TCR statistical data.

\begin{tabular}{lrrrrr}
\hline & \multicolumn{2}{c}{ BCR } & & \multicolumn{2}{c}{ TCR } \\
\cline { 2 - 3 } \cline { 5 - 6 } Sequence numbers & PTC & Pericarcinoma & & PTC & Pericarcinoma \\
\hline Total reads number & 486,007 & 745,353 & & 841,591 & 473,761 \\
Immune sequences number & 455,365 & 714,856 & & 822,485 & 462,413 \\
Unknown sequences number & 30,642 & 30,497 & & 19,106 & 11,348 \\
Productive sequences number & 386,246 & 613,972 & 579,158 & 343,331 \\
Nonproductive sequences number & 69,119 & 100,884 & 243,327 & 119,082 \\
In-frame sequences number & 403,093 & 636,692 & & 600,620 & 359,473 \\
Out-of-frame sequences number & 51,364 & 76,997 & 215,049 & 98,430 \\
Total CDR3 sequences number & 292,683 & 341,209 & & 561,607 & 335,334 \\
Unique CDR3 nt sequences number & 13,896 & 27,156 & & 17,612 & 20,410 \\
Unique CDR3 aa sequences number & 10,763 & 21,799 & 13,669 & 16,789 \\
\hline
\end{tabular}

BCR, B-cell receptors; TCR, T-cell receptors; PTC, papillary thyroid carcinoma; CDR, complementarity-determining region; nt, nucleotide; aa, amino acid.
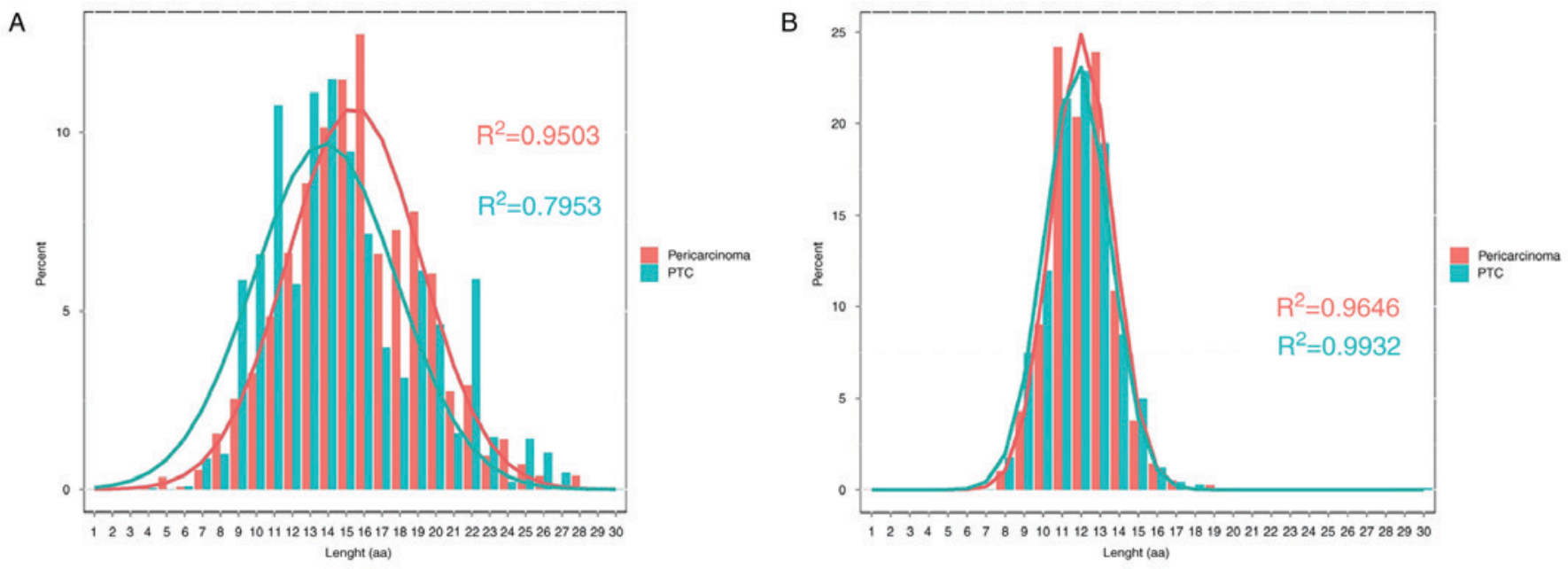

Figure 1. Length distribution of (A) immunoglobulin heavy chain and (B) T-cell receptor $\beta$ chain complementarity-determining region 3 in the PTC group $(n=5)$ and corresponding pericarcinoma group $(n=5) . R^{2}$ is Gaussian distribution fitting value, and the closer the value to 1 , the closer the result to normal distribution. Different colors represent different groups, as shown in the illustration. PTC, papillary thyroid carcinoma.

amino acid level in PTC (Fig. 3A). The unique TRB nucleotide clonotypes accounted for $5.50 \%$ at the nucleotide level and $7.29 \%$ at the amino acid level in the PTC group (Fig. 3B). Overall, the common sequences of the PTC group were higher than the matched surrounding tissue, the extent of the common clonotype expressions of the amino acid was slightly higher than the nucleotide level.

IGH and TRB CDR3 sequence diversity and HECs Shannon entropy. Shannon entropy is a measure of immune diversity that takes richness and evenness into account. The index is between 0.0 and 1.0. The closer to 1 the higher the immune diversity of the individual, and the closer to zero the worse the immune diversity of the individual (14). The immune diversity of PTC tissues demonstrated a reduction compared with pericarcinoma tissues (Table II). The reduction of immune repertoire diversity is an important immune system characteristic of patients with PTC. In the process of tumor invasion, immune function is in a low state relative to normal function.

Here, clones with a frequency of $>0.5 \%$ of the analyzed BCR and TCR sequences were defined as HECs. The number of HECs in PTC was higher compared with that of the corresponding pericarcinoma group.

Comparison of the expression levels of different clones and shared clones between PTC and pericarcinoma. According to the aforementioned research results, considering the diminishment of immune repertoire diversity may be associated with the increase or decrease of a number of clonings, the unique clonetype frequency distribution was then tested. All the unique clonetype were divided into four different groups, $0.1-1.0,0.01-0.10,0.001-0.010$ and $0.000-0.001 \%$. The highest clone frequency percentage of IGH was at a $0.1-1.0 \%$ degree 

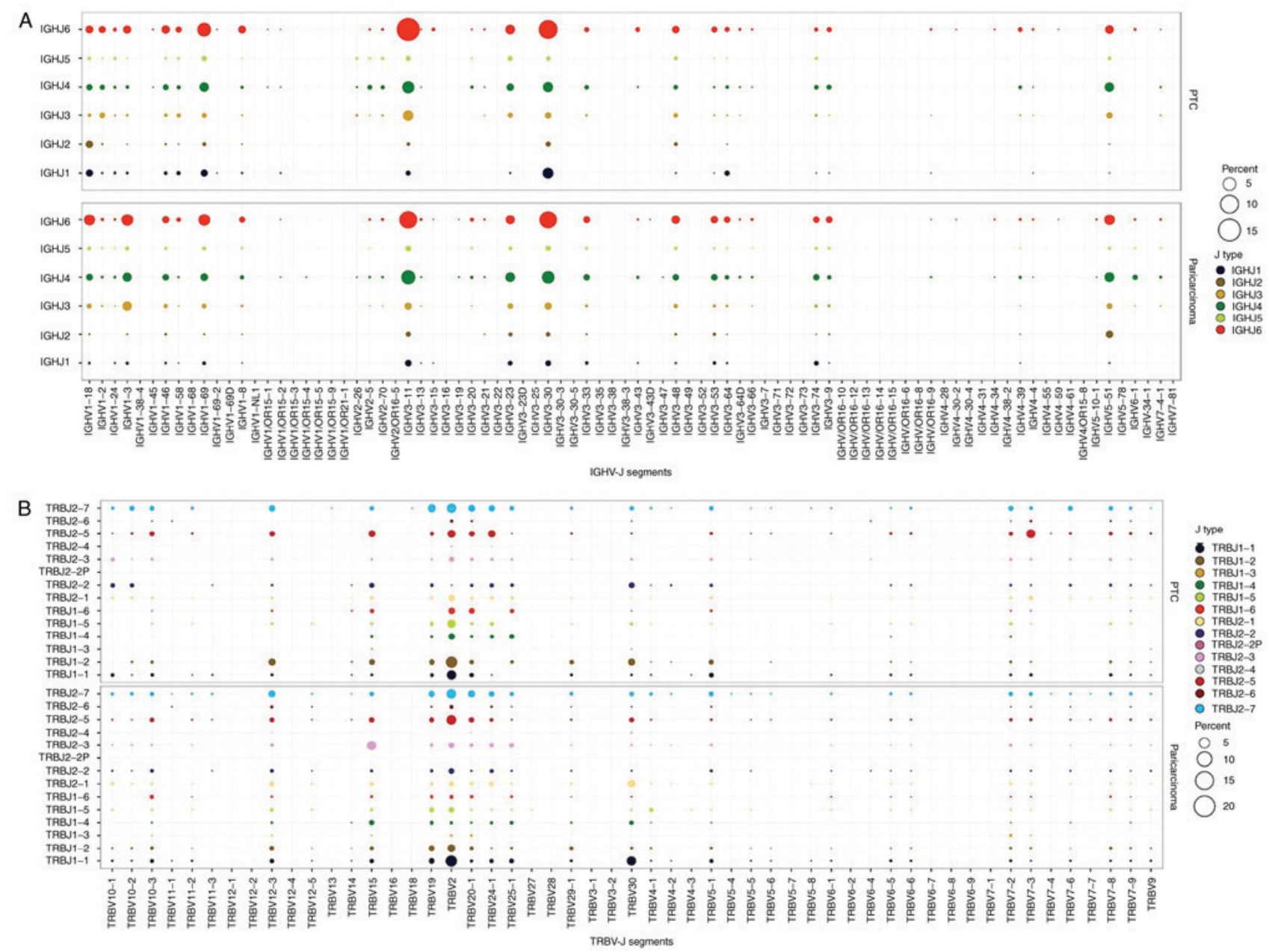

Figure 2. V-J region gene usage of IGH and TRB from the PTC group and corresponding pericarcinoma group. (A) IGHV-J segments and (B) TRBV-J segments. IGH, immunoglobulin heavy chain; TRB, T-cell receptor $\beta$ chain; PTC, papillary thyroid carcinoma.

A
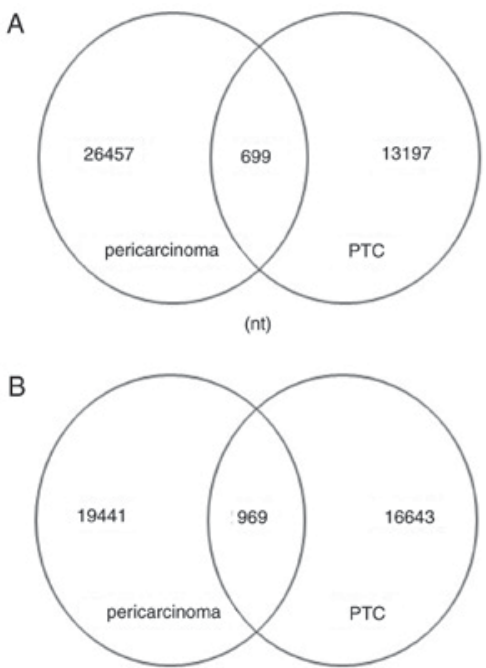

(nt)

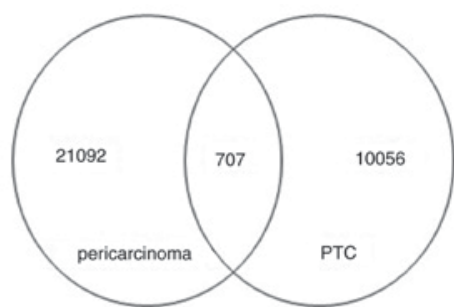

(aa)

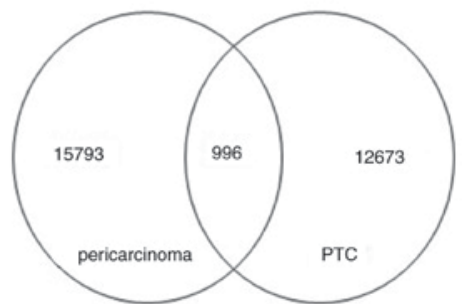

(aa)

Figure 3. Proportion of common nucleotide clonotypes and amino acid clonotypes and of (A) the immunoglobulin heavy chain CDR3 sequence and (B) the T-cell receptor $\beta$ chain CDR3 sequence in the PTC and corresponding pericarcinoma group. The Venn diagrams present the total number of nucleotide clonotypes and amino acid clonotypes in the two groups. The numbers of specical CDR3 clonotypes are shown in the nonoverlapping sections, and the numbers of shared CDR3 clonotypes are indicated in the relevant overlapping areas. CDR, complementarity-determining region; PTC, papillary thyroid carcinoma.

of expansion, and the unique cloning frequency distribution accounted for $80.92 \%$ in PTC tissues, whilst it only contributed
47.14\% in pericarcinoma tissues (Fig. 4A). A total of 42 HECs in PTC were obtained, but only 8 in pericarcinoma. The 
Table II. Diversity and highly expended clone statistics.

\begin{tabular}{|c|c|c|c|c|}
\hline \multirow[b]{2}{*}{ Diversity index } & \multicolumn{2}{|c|}{$\mathrm{BCR}$} & \multicolumn{2}{|c|}{ TCR } \\
\hline & PTC & Pericarcinoma & PTC & Pericarcinoma \\
\hline Highly expended clone number all & 42 & 8 & 28 & 8 \\
\hline Highly expended clone ratio all & 0.499906042 & 0.08306639 & 0.224917068 & 0.138205491 \\
\hline Shannon entropy all & 0.46214436 & 0.58059448 & 0.492826469 & 0.577329276 \\
\hline
\end{tabular}

BCR, B-cell receptors; TCR, T-cell receptors; PTC, papillary thyroid carcinoma.
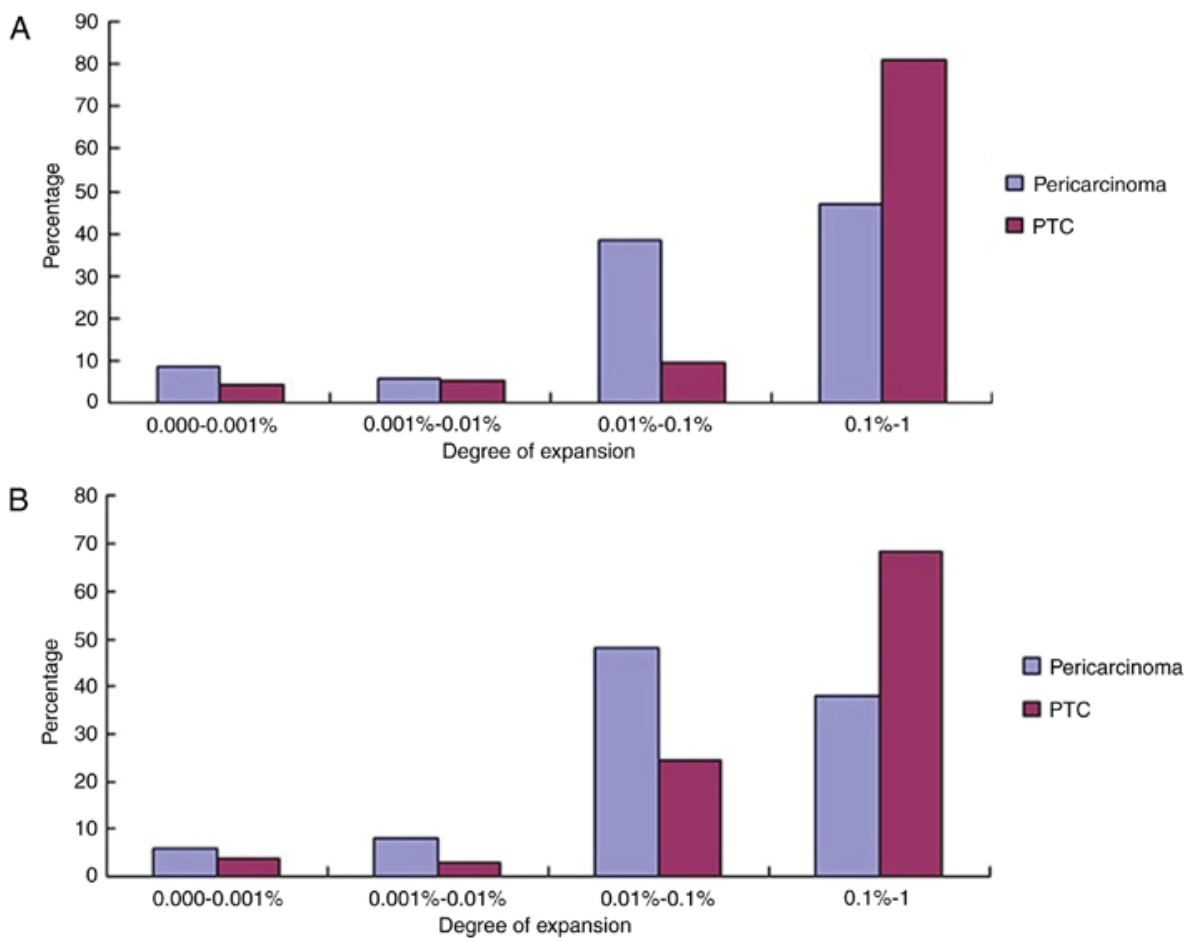

Figure 4. Degree of expansion and frequency distribution of (A) B-cell clones and (B) T-cell clones. The frequency distribution revealed that the majority of the repertoire, which comprised of a small number of highly expended clones, presented a right-skewed distribution, i.e., the majority of the clones were high frequency. The $\mathrm{x}$-axis depicts the degree of expansion; the $\mathrm{y}$-axis depicts the percentage of each clone frequency. PTC, papillary thyroid carcinoma.

highest clone frequency percentage of TRB was at a $0.1-1.0 \%$ degree of expansion, and the unique cloning frequency distribution accounted for $68.43 \%$ in PTC (Fig. 4B), whilst it only contributed $38.00 \%$ in pericarcinoma. A total of $28 \mathrm{HECs}$ were obtained in PTC, but only 8 in pericarcinoma similar to the HECs in IGH.

Whether in IGH or TRB CDR3 sequences, the numbers of the expression cloning of HECs in PTC were increased compared with pericarcinoma. Furthermore, as presented in Fig. 5, there was no shared clone of HECs in the two groups either at the nucleotide or at the amino acid expression level. There were 32 and 3 shared HECs of IGH CDR3 nucleotide sequences and 31 and 3 shared HECs of IGH CDR3 amino acid sequences in PTC and pericarcinoma groups, respectively. There were additionally 28 and 5 shared HECs of TRB CDR 3 nucleotide sequences and 28 and 5 shared HECs of TRB CDR3 amino acid sequences in PTC and pericarcinoma groups, respectively.

\section{Discussion}

The human adaptive immune system is composed of $\mathrm{B}$ and $\mathrm{T}$ cell receptors that bind to non-pathogenic or pathogen derived antigens, which provides protection against an enormous variety of pathogens and non-pathogens (7). Additionally, the immune repertoire diversity may be affected by lymphocyte cell clonal expansion following the occurrence and development of PTC, so that it affects the immune response of patients to the pathogen $(2,8)$. CDR3 is the most variable and directly determines the antigen-binding specificity of the BCR and TCR (15). In the present study, HTS was used in order to investigate the characteristics and polymorphisms of the IGH and TRB CDR3 gene from PTC and corresponding pericarcinoma tissues from 5 patients. The distributions of CDR3 length among 292,683 and 341,209 total IGH CDR3 sequences and 561,607 and 335,334 total TRB CDR3 sequences from PTC and corresponding pericarcinoma, 

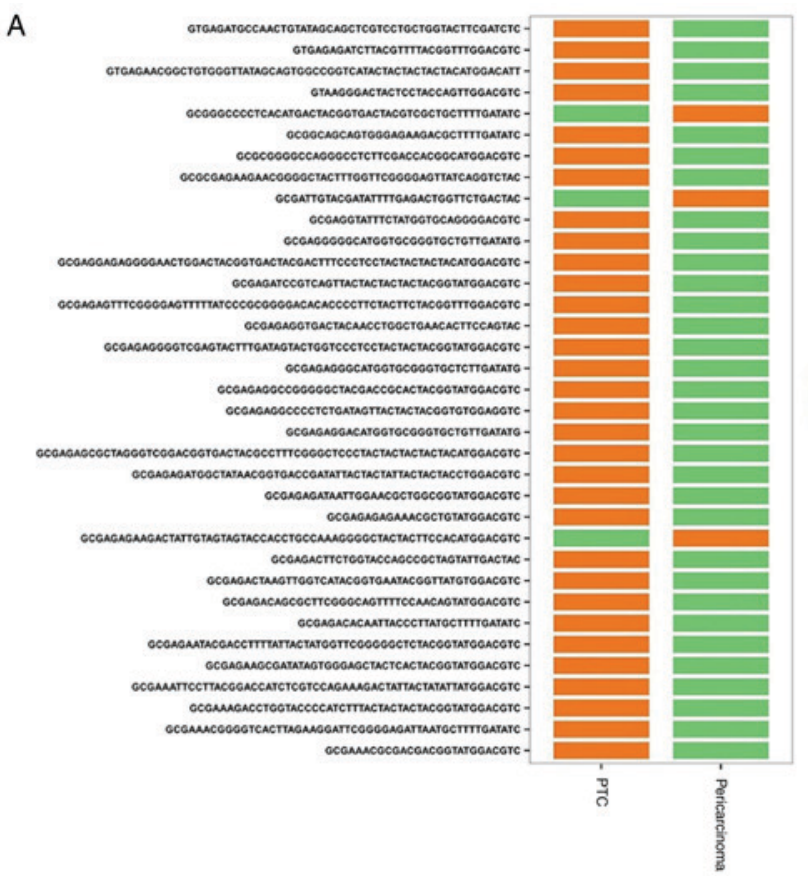

B

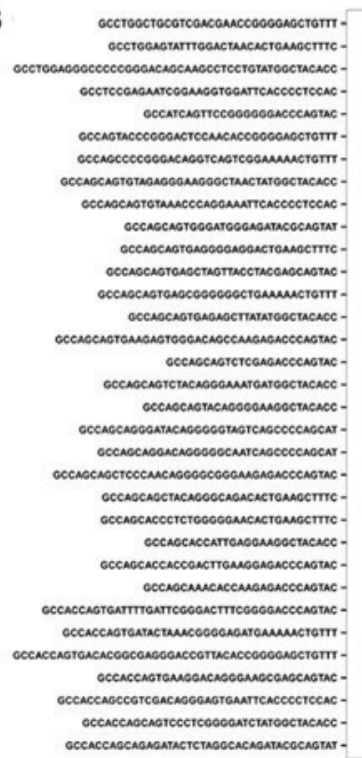

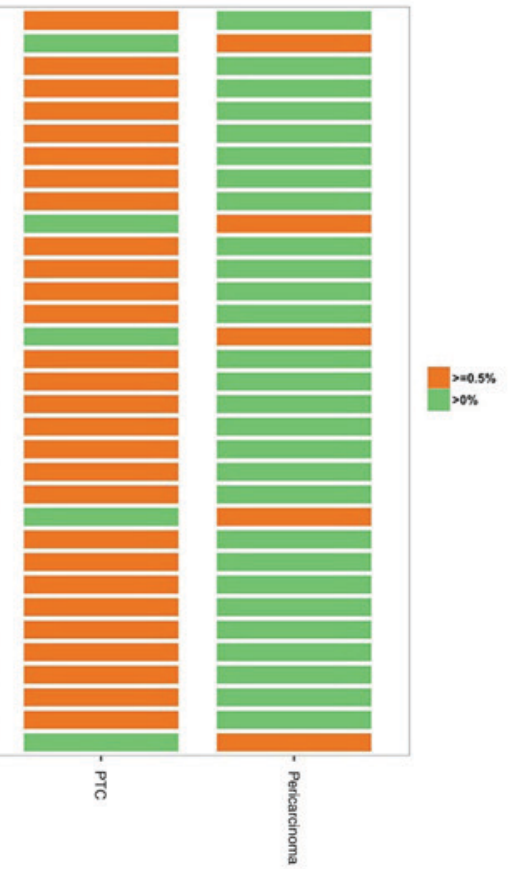
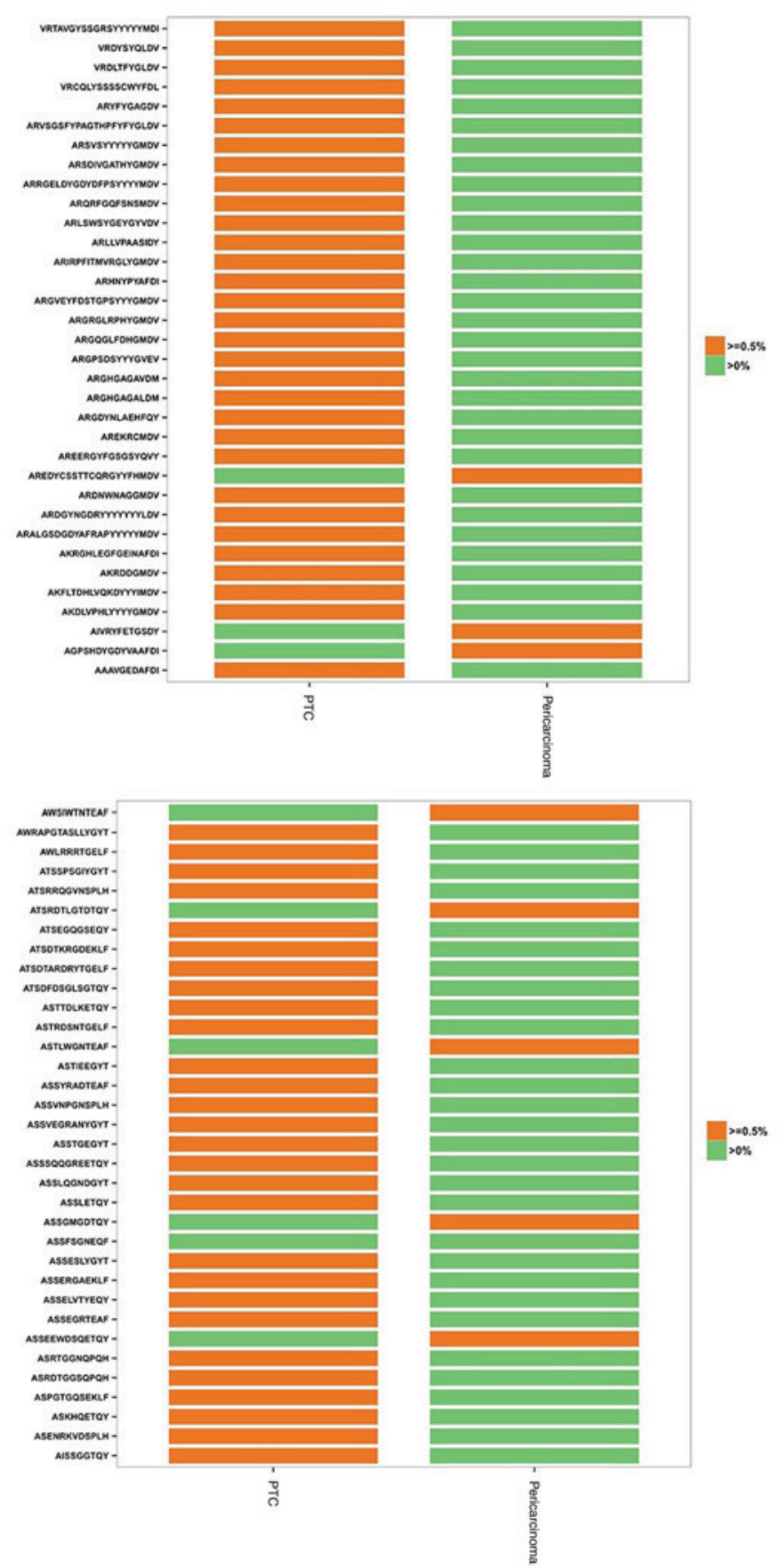

Figure 5. Shared HECs between PTC and corresponding pericarcinoma groups at the nt and aa level. (A) A total of 32 and 3 shared HECs of IGH CDR3 nt sequences from PTC and corresponding pericarcinoma groups, respectively, were on the left, and a total of 31 and 3 shared HECs of IGH CDR3 aa sequences from PTC and corresponding pericarcinoma groups, respectively, were on the right. (B) A total of 28 and 5 shared HECs of TRB CDR3 nt sequences from PTC and corresponding pericarcinoma groups, respectively, were on the left, and a total of 28 and 5 shared HECs of TRB CDR3 aa sequences from PTC and corresponding pericarcinoma groups, respectively, were on the right. HEC, highly expended clones; PTC, papillary thyroid carcinoma; IGH, immunoglobulin heavy chain; CDR, complementarity-determining region; TRB, T-cell receptor $\beta$ chain; nt, nucleotide; aa, amino acid.

respectively, were evaluated. The most frequently observed CDR3 lengths were identified. The difference of IGH CDR3 length distributions between PTC and pericarcinoma groups was identified. However, the difference of TRB between the two groups was minimal. It may result in a delay in the expression of CDR3 length distributions of TRB region and it was different compared with that of TRBV families derived from pleural effusion, which exhibited a predominance of a selected CDR3 length (16). Hou et al (12) detected that the specific skewed usage of TRBV segments may be associated with the susceptibility to unexplained pregnancy loss. The aforementioned study indicated that the CDR3 length distribution may be associated with the disease. The goal is to identify, quantify and statistically assess differences between repertoires so as to offer a better diagnostic or predictive tool for pathologies involving the immune system (17).

During B and T lymphocyte development, V, D and J gene segments are rearranged through targeted DNA recombination. Through this method, a variable CDR3 region with a highly diversity may be formed $(7,8)$. The usage frequency 
of $\mathrm{V}, \mathrm{D}, \mathrm{J}$ and $\mathrm{V}-\mathrm{J}$ regions were systematically analyzed. A total of $83 \mathrm{~V}$ genes, $37 \mathrm{D}$ genes and $6 \mathrm{~J}$ genes of IGH CDR3 sequences and $58 \mathrm{~V}$ genes, $2 \mathrm{D}$ genes and $14 \mathrm{~J}$ genes of TRB CDR3 sequences were obtained. The upregulated expressions and downregulated expressions of IGH and TRB V genes in the two groups were identified. The quantity of upregulated expression was greater compared with that of the downregulated expression in IGH genes. No differences existed in the D genes of IGH and TRB CDR3 regions between two groups. Open reading frame expression was mostly observed in IGHD genes, compared with TRBD genes. The TRBJ genes usage ratio in two groups presented a similar pattern as IGHJ genes. These results indicate that the immune system of patients was active. To better understand the binding specificity of the immune system, $275 \mathrm{IGHV}$-J pairings and $536 \mathrm{TRBV}-\mathrm{J}$ pairings were identified in PTC and pericarcinoma groups. It has been revealed that the $\mathrm{N}$-glycosylation of IGHV regions including IGHV4-34 may confer a selective advantage via interactions of the glycosylated BCR with mannose binding lectins in the GC and thus help account for the prevalence of IGHV4-34 usage in follicular lymphoma $(18,19)$. In the present study, IGHV3-11/IGHJ6, TRBV2/TRBJ1-2 and TRBV2/TRBJ1-1 may be the biomarkers expressed during PTC development.

Amino acid sequences of CDR3, which reveal the function of the immune repertoire, may reflect the specificity of the combination of lymphocytes and antigens compared with the $\mathrm{V}-\mathrm{J}$ gene. Therefore, it is worth exploring the shared sequences at the amino acid and nucleotide level. The aforementioned data analysis revealed that the extent of the common clonotype expressions at the amino acid level was slightly higher compared with the nucleotide level.

CDR3 sequences, which determine a unique BCR and TCR clone, may detect a lymphocyte clone. The index of PTC Shannon entropy (20) and HECs between the two groups are different. The diversity demonstrated a reduction in PTC compared with pericarcinoma groups, and HECs in PTC tissues were higher compared with that of corresponding pericarcinoma tissues. Brezinschek et al (21) reported that $\mathrm{B}$ cells underwent clonal expansion in response to chronic stimulation, which may conceivably be due to pathogens or autoantigens. The identification of the Ag or Ags against which this selective B cell response occurs may provide notable insights into the mechanism of blood-brain barrier disruption, immune-mediated demyelination and atrophy (22). The cellular adaptive immune system mounts a response to numerous different solid tumor types, mediated by tumor infiltrating $\mathrm{T}$ lymphocytes (23). The findings of a previous study revealed increased TCR diversity in prostate tumor types following treatment with Sipuleucel-T, suggesting that active immunotherapies elicit measurable changes within the tumor microenvironment, and increasing TCR diversity may be positive (24). Clemente et al (25), studying large granular lymphocyte leukemia with similar techniques, revealed a low diversity of TCR species at the baseline, suggesting that growing tumors may be characterized by a low diversity, and/or non-functional and infiltrating $\mathrm{T}$ cells.

To ensure efficient protection of the organism, the immune system combines two conflicting needs: A recognition of a wide array of antigens and an efficient and timely response (26). The expression levels of different clones and shared clones between
PTC and pericarcinoma groups were compared. The highest clone frequency percentage of immune repertoire were at a $0.1-1.0 \%$ degree of expansion and the expression numbers of HECs were higher in PTC compared with the matched group. However, the amplification extent of HECs was reduced in the 0.01-0.1\% degree of expansion group in PTC. This may reveal the degree of expansion and the frequency distribution of the predominant changes of $\mathrm{B}$ and $\mathrm{T}$ cell clones in the process of the tumor invasion. Furthermore, there was no shared clone of HECs in the two groups either at the nucleotide or at the amino acid expression level. Relevant to this investigation, Emerson et al (23) revealed a number of overlaps between tumor and blood, primarily tumor TCRs were observed only in tumor tissue. This suggests that the intratumoral microenvironment is characterized by a distinct but internally homogeneous $\mathrm{T}$ cell repertoire that may potentially be manipulated through the development of immunotherapy.

In conclusion, previous studies in tumor immunology have highlighted the need for simple, quantitative and reproducible measures of the host anti-tumor immune response to improve prognosis (i.e., tumor staging that includes immunological considerations) and to predict the response of patients to immunotherapy (27-29). Analysis of immune repertoires by such an approach may be valuable in a clinical setting, for prognostic potential analysis and the measurement of clinical responses to therapy. However, in the present study, there was no significance identified due to the small sample numbers. The genetic basis of drug resistance, pathogenesis, persistence and latency may be identified through analysis with a larger sample size.

As the cost of HTS declines, it is expected that direct HTS of the immune repertoire may provide more useful information for assessing the immune repertoire size, diversity, cloning tracking and find public clone in B-cell and T-cell populations in the development of PTC.

\section{Acknowledgements}

Not applicable.

\section{Funding}

The present study was supported by the National Nature Science Foundation of China (grant nos. 30170050, 81402370 and 81671596), Guangdong Natural Science Foundation (grant no. 2015A030313829), the Scientific and Technology Foundation of Guangdong (grant nos. 2015B090904007 and 2016A050503009), the Natural Science Foundation of Guangdong (grant no. 2016A030313029), the Scientific Plan Program of Guangdong (grant no. 2014A020212038), the Innovation Program of Shenzhen (grant no. JCYJ20150330102720122), the International Cooperation Foundation of Shenzhen (grant no. GJHZ20160301163138685) and the Pingshan New District Incubation Foundation of Shenzhen (grant no. 201501).

\section{Availability of data and materials}

The datasets used or analyzed during the current study are available from the corresponding author on reasonable request. 


\section{Authors' contributions}

All authors reviewed and approved the manuscript prior to the submission. GS and WP conceived and designed the experiments. PZ and JZ performed the experiments. JQ and SL analyzed the data. CZ and NX interpreted the patient data regarding the papillary thyroid carcinoma. ZQ performed the pathological examination of the papillary thyroid. YD conceived and designed the experiments, analyzed the data. LQ interpreted the data and drafted the manuscript. All authors read and approved the final manuscript.

\section{Ethics approval and consent to participate}

Written informed consent for participation in the present study was obtained from all participants. The present study was approved by the Ethics Committee of Shenzhen People's Hospital and abided by the Helsinki Declaration for medical research involving human subjects.

\section{Consent for publication}

Written informed consent for participation in the present study was obtained from all subjects. All participants provided consent for publication of data in research and press articles, and websites, exclusively for non-profit-making purposes.

\section{Competing interests}

The authors declare that they have no competing interests.

\section{References}

1. Jung CW, Kong JS, Seol H, Park S, Koh JS, Lee SS, Kim MJ, Choi IJ and Myung JK: Expression of activated Notch1 and Hey1 in papillary thyroid carcinoma. Histopathology 70: 301-308, 2017

2. Georgiades F, Vasiliou G, Kyrodimos E and Thrasyvoulou G: Extensive laryngeal infiltration from a neglected papillary thyroid carcinoma: A case report. World J Clin Cases 4: 187-190, 2016.

3. Chen W, Zheng R, Zeng H, Zhang S and He J: Annual report on status of cancer in China, 2011. Chin J Cancer Res 27: 2-12, 2015

4. Zhu H, Lv Z, An C, Shi M, Pan W, Zhou L, Yang W and Yang M: Onco-lncRNA HOTAIR and its functional genetic variants in papillary thyroid carcinoma. Sci Rep 6: 31969, 2016

5. Wang T, Blumer I, Boerner S and Asa SL: Synchronous papillary carcinoma of thyroid and lung. Endocr Pathol 27: 268-270, 2016

6. Moritani S: Parapharyngeal metastasis of papillary thyroid carcinoma. World J Surg 40: 350-355, 2016.

7. Calis JJ and Rosenberg BR: Characterizing immune repertoires by high throughput sequencing: Strategies and applications. Trends Immunol 35: 581-590, 2014.

8. Schanz M,Liechti T,Zagordi O, Miho E, Reddy ST, Günthard HF, Trkola A and Huber M: High-throughput sequencing of human immunoglobulin variable regions with subtype identification. PloS One 9: e111726, 2014.

9. Quail MA, Smith M, Coupland P, Otto TD, Harris SR, Connor TR, Bertoni A, Swerdlow HP and Gu Y: A tale of three next generation sequencing platforms: Comparison of ion torrent, pacific biosciences and illumina Miseq sequencers. BMC Genomics 13: 341, 2012.

10. Reuter JA, Spacek DV and Snyder MP: High-throughput sequencing technologies. Mol Cell 58: 586-597, 2015

11. Lefranc MP, Giudicelli V, Ginestoux C, Bodmer J, Müller W, Bontrop R, Lemaitre M, Malik A, Barbié V and Chaume D: IMGT, the international ImMunoGeneTics database. Nucleic Acids Res 27: 209-212, 1999.
12. Hou X, Lu C, Chen S, Xie Q, Cui G, Chen J, Chen Z, Wu Z, Ding Y, Ye P, et al: High throughput sequencing of $\mathrm{T}$ cell antigen receptors reveals a conserved TCR repertoire. Medicine (Baltimore) 95: e2839, 2016.

13. Sui W, Hou X, Zou G, Che W, Yang M, Zheng C, Liu F, Chen P, Wei X, Lai L and Dai Y: Composition and variation analysis of the TCR $\beta$-chain CDR3 repertoire in systemic lupus erythematosus using high-throughput sequencing. Mol Immunol 67: 455-464, 2015

14. Yu X, Almeida JR, Darko S, van der Burg M, DeRavin SS, Malech H, Gennery A, Chinn I, Markert ML, Douek DC and Milner JD: Human syndromes of immunodeficiency and dysregulation are characterized by distinct defects in T-cell receptor repertoire development. J Allergy Clin Immunol 133: 1109-1115, 2014.

15. Robins H: Immunosequencing: Applications of immune repertoire deep sequencing. Curr Opin Immunol 25: 646-652, 2013.

16. Li D, Gao G, Li Z, Sun W, Li X, Chen N, Sun J and Yang Y: Profiling the T-cell receptor repertoire of patient with pleural tuberculosis by high-throughput sequencing. Immunol Lett 162 : 170-180, 2014.

17. Miqueu P, Guillet M, Degauque N, Doré JC, Soulillou JP and Brouard S: Statistical analysis of CDR3 length distributions for the assessment of T and B cell repertoire biases. Mol Immunol 44: 1057-1064, 2007

18. Zhu D, McCarthy H, Ottensmeier CH, Johnson P, Hamblin TJ and Stevenson FK: Acquisition of potential N-glycosylation sites in the immunoglobulin variable region by somatic mutation is a distinctive feature of follicular lymphoma. Blood 99: 2562-2568, 2002.

19. Radcliffe CM, Arnold JN, Suter DM, Wormald MR, Harvey DJ, Royle L, Mimura Y, Kimura Y, Sim RB, Inogès S, et al: Human follicular lymphoma cells contain oligomannose glycans in the antigen-binding site of the B-cell receptor. J Biol Chem 282: 7405-7415, 2007

20. Nemzer LR: Shannon information entropy in the canonical genetic code. J Theor Biol 415: 158-170, 2017.

21. Brezinschek HP, Foster SJ, Brezinschek RI, Dörner T, Domiati-Saad R and Lipsky PE: Analysis of the human VH gene repertoire. Differential effects of selection and somatic hypermutation on human peripheral CD5(+)/IgM+ and CD5(-)/IgM+ B cells. J Clin Invest 99: 2488-2501, 1997.

22. Baranzini SE, Jeong MC, Butunoi C, Murray RS, Bernard CC and Oksenberg JR: B cell repertoire diversity and clonal expansion in multiple sclerosis brain lesions. J Immunol 163 : 5133-5144, 1999.

23. Emerson RO, Sherwood AM, Rieder MJ, Guenthoer J, Williamson DW, Carlson CS, Drescher CW, Tewari M, Bielas JH and Robins HS: High-throughput sequencing of T-cell receptors reveals a homogeneous repertoire of tumour-infiltrating lymphocytes in ovarian cancer. J Pathol 231: 433-440, 2013.

24. McNeel DG: TCR diversity-a universal cancer immunotherapy biomarker? J Immunother Cancer 4: 69, 2016.

25. Clemente MJ, Przychodzen B, Jerez A, Dienes BE, Afable MG, Husseinzadeh H, Rajala HL, Wlodarski MW, Mustjoki S and Maciejewski JP: Deep sequencing of the T-cell receptor repertoire in CD8+ T-large granular lymphocyte leukemia identifies signature landscapes. Blood 122: 4077-4085, 2013.

26. Arstila TP, Casrouge A, Baron V, Even J, Kanellopoulos J and Kourilsky P: A direct estimate of the human alphabeta $\mathrm{T}$ cell receptor diversity. Science 286: 958-961, 1999.

27. Ascierto PA, Capone M, Urba WJ, Bifulco CB, Botti G, Lugli A, Marincola FM, Ciliberto G, Galon J and Fox BA: The additional facet of immunoscore: Immunoprofiling as a possible predictive tool for cancer treatment. J Transl Med 11: 54, 2013.

28. Galon J, Pagès F, Marincola FM, Thurin M, Trinchieri G, Fox BA, Gajewski TF and Ascierto PA: The immune score as a new possible approach for the classification of cancer. J Transl Med 10: 1, 2012.

29. Galon J, Angell HK, Bedognetti D and Marincola FM: The continuum of cancer immunosurveillance: Prognostic, predictive, and mechanistic signatures. Immunity 39: 11-26, 2013

(i) (-) This work is licensed under a Creative Commons Attribution-NonCommercial-NoDerivatives 4.0 International (CC BY-NC-ND 4.0) License. 